\title{
Importancia y beneficios del consumo de huevo de gallina enriquecido con selenio: revisión narrativa
}

\author{
Importance and benefits of selenium-enriched hen egg intake: \\ A narrative review \\ Importância e benefícios do consumo de ovos de galinha enriquecidos com selénio: \\ uma narrativa de revisão
}

Jorge H. Ulloa ${ }^{1,2^{*}}$, Sebastián Cifuentes ${ }^{1,2}$, Valentín Figueroa', Emelyn Van Uden², Susana Tafur².

Recibido: 21 de noviembre de 2020. Aceptado para publicación:1 de marzo de 2021.

Publicado en línea: 5 de marzo de 2021.

https://doi.org/10.35454/rncm.v4n3.238

\begin{abstract}
Resumen
Introducción: el selenio ha demostrado poseer efectos benéficos sobre la salud humana, entre los que destacan el fortalecimiento del sistema inmune, efectos antiinflamatorios y disminución de la lipoxidación. Además, su deficiencia puede ocasionar enfermedades como la enfermedad de Keshan y de Kashin-Beck, ambas con una mortalidad del $50 \%$, aproximadamente. El objetivo de este artículo es identificar y sintetizar las funciones y ventajas del consumo de huevo enriquecido con selenio en la salud humana.

Métodos: se realizó una revisión bibliográfica tanto en español como en inglés en las bases de datos de Google Scholar y Pubmed. Se emplearon los términos "huevo", "selenio" y "síndrome metabólico", y se tomaron en consideración aquellos artículos con textos completos disponibles y con relevancia en cuanto a la relación entre el huevo y el selenio.
\end{abstract}

Resultados: se ha evidenciado que el selenio es esencial en nuestra dieta y presenta numerosos efectos benéficos sobre la salud. Actualmente, las tendencias nutricionales van dirigidas no solo a cubrir las necesidades básicas de selenio, sino también a la prevención y tratamiento de enfermedades en humanos. El huevo es un alimento de fácil acceso, bajo costo y de gran aporte nutricional con baja carga calórica, por lo que su enriquecimiento

\section{Summary}

Introduction: Selenium has been shown to have beneficial effects on human health, such as strengthening of the immune system, anti-inflammatory effects and decreased lipoxidation. Moreover, its deficiency can cause diseases such as Keshan disease and Kashin-Beck disease, which have a mortality of approximately $50 \%$. The objective of this article is to identify and summarize the functions and advantages of consuming seleniumenriched egg on human health.

Methods: A literature review was carried out in Spanish and English in Google Scholar and PUBMED databases using the terms "egg", "selenium", and "metabolic syndrome". Only full text articles relevant to the relationship between egg and selenium were taken into consideration.

Results: It has been shown that selenium is essential in our diet and has numerous beneficial effects on health. Currently, nutritional trends are aimed at not only meeting the basic needs in selenium, but also to the prevention and treatment of diseases in humans. Eggs are an easily accessible, low-cost food with a high nutritional contribution and a low caloric load. Therefore, its enrichment with selenium could guarantee an adequate supply in most of the Colombian population.

Conclusion: The enrichment of hen eggs with selenium would not only help

\section{Resumo}

Introdução: O selênio tem demonstrado ter efeitos benéficos na saúde humana, entre os quais destaca-se o fortalecimento do sistema imunológico, efeitos antiinflamatórios e diminuição da lipoxidação. No entanto, sua deficiência pode causar doenças como a doença de Keshan e a doença de Kashin-Beck, ambas com mortalidade de aproximadamente $50 \%$. O objetivo deste artigo é identificar e sintetizar as funções e vantagens do consumo de ovo enriquecido com selênio.

Métodos: Foi realizada uma revisão bibliográfica em espanhol e inglês nas bases de dados de Google Scholar e PUBMED. Foram utilizados os termos "ovo", "selênio", "síndrome metabólica", foram considerados aqueles artigos com textos completos disponíveis e relevantes no referente à relação entre o ovo e o selênio.

Resultados: tem sido evidenciado que o selênio é essencial em nossa dieta e tem inúmeros efeitos benéficos na saúde. Atualmente, as tendências nutricionais estão direcionadas não apenas para cobrir as necessidades básicas de selênio, mas também para a prevenção e tratamento de doenças em humanos. $O$ ovo é um alimento de fácil acesso, baixo custo e alto aporte nutricional com baixa carga calóri$\mathrm{ca}$, de modo que seu enriquecimento com selênio poderia garantir uma substituição 
con selenio podría garantizar una adecuada suplencia en la mayoría de la población colombiana.

Conclusión: el enriquecimiento del huevo de gallina con selenio no solo ayudaría a prevenir enfermedades, sino que también representaría una medida de prevención de enfermedades y promoción de la salud.

Palabras clave: huevo, selenio, salud, síndrome metabólico. prevent diseases but would also represent a measure of health promotion and prevention.

Keywords: Egg; Selenium; Health; Metabolic Syndrome. adequada na maioria da população colombiana.

Conclusão: $O$ enriquecimento do ovo de galinha com selênio não só ajudaria a prevenir doenças, mas também representaria uma medida de promoção e prevenção à saúde.

Palavras-chave: ovo, selênio, saúde, síndrome metabólica.
Sección de Cirugía Vascular, Hospital Universitario Fundación Santa Fe, Bogotá, Colombia.

2 Facultad de Medicina de la Universidad de los Andes, Bogotá, Colombia.
*Correspondencia: Jorge Hernando Ulloa. cirugiavascular@yahoo.com

\section{INTRODUCCIÓN}

El huevo representa una fuente de alimento con amplio aporte nutricional, económica y de fácil acceso para la gran mayoría de la población. De igual forma, su producción tiene un impacto ambiental significativamente menor comparado con otras fuentes de proteína animal. Los estudios han demostrado que las recomendaciones dadas en décadas pasadas sobre la restricción de su consumo por los niveles de colesterol no son válidas. Actualmente, hay evidencia de que no existe una relación significativa entre el consumo de huevo y el aumento del colesterol en la sangre. Se ha demostrado que incluso el consumo de 7 huevos por semana es sano y recomendable ${ }^{(1)}$.

$\mathrm{Su}$ aporte nutricional lo convierte en una excelente opción de alimentación para individuos con requerimientos nutricionales especiales tales como niños, ancianos y mujeres gestantes. Uno de los elementos con los que se enriquece el huevo habitualmente es el selenio, un elemento con funciones fisiológicas importantes en el cuerpo humano. Su deficiencia aumenta el riesgo de mortalidad y compromete el sistema inmune; por otra parte, es esencial en la reproducción humana y reduce el riesgo de enfermedad tiroidea autoinmune ${ }^{(2)}$. Por último, varios estudios se han llevado a cabo para explorar las propiedades anticancerígenas, antiinflamatorias y su potencial efecto en la prevención de enfermedad cardiovascular ${ }^{(3)}$.

El objetivo de este artículo es identificar y sintetizar las funciones y ventajas del consumo de huevo enriquecido con selenio. De esta manera, se pretende incrementar la evidencia que respalde el consumo de este alimento y su inclusión en la dieta diaria de una población como la colombiana, dada su accesibilidad económica y sus beneficios en la salud humana.

\section{ANÁLISIS Y DISCUSIÓN}

\section{Selenio}

El selenio es un oligoelemento esencial no metálico que se requiere para la síntesis de selenocisteína. Esta es esencial en la síntesis de selenoproteínas las cuales se pueden presentar en 2 formas: orgánica e inorgánica ${ }^{(4,5)}$. El selenio es crucial tanto para los animales como para los humanos. Según estudios realizados, se cree que el selenio orgánico como adición en los alimentos se absorbe mejor por los animales y humanos ${ }^{(5,6)}$. Las principales formas dietéticas son la selenocisteína, derivada de alimentos de origen animal, y la selenometionina (SeMet), que se obtiene de alimentos de origen animal y productos de cereales de áreas con suelo ricos en selenio ${ }^{(4,6-8)}$. Independientemente de la distribución del selenio en el suelo, hay otros factores que afectan su concentración, como: el pH del suelo, la lluvia, el contorno de la tierra y la actividad microbiana ${ }^{(4,9)}$. La SeMet se encuentra predominantemente en granos, pescado (mariscos), carne, aves, productos lácteos y huevos $^{(6,10)}$; por otra parte, las verduras como nabos, guisantes, fríjoles, zanahorias, tomates, remolachas, papas y pepinos contienen un máximo de $6 \mathrm{mg} / \mathrm{g}$ de selenio. Algunas verduras como la cebolla y los espárragos pueden acumular hasta $17 \mu \mathrm{g} / \mathrm{g}$ de selenio cuando se cultivan en suelos con abundante selenio ${ }^{(7)}$. 


\section{Selenio en el huevo}

El contenido de selenio en la comida depende del alimento y la riqueza del suelo de donde se consiga ${ }^{(4,6-8,}$ ${ }^{11,12)}$. Los alimentos de origen animal son una mejor fuente dietética de selenio, esto debido a que es un nutriente esencial para los animales y la alimentación de estos es complementada con selenio ${ }^{(7,11)}$. La cantidad y la forma (orgánica o inorgánica) del selenio encontrado en los huevos de gallina dependerá del nivel de suplencia de este en la dieta de las gallinas. La cantidad de SeMet que aportará un huevo en la dieta se relaciona con las proteínas de la clara del huevo originadas en el oviducto; por otra parte, la cantidad de selenito estará relacionado con las proteínas de la yema de huevo que se sintetizan en el hígado. La suplementación de selenio en la dieta de las aves podrá reflejarse en el incremento de selenio en la yema del huevo, lo cual actuará como un antioxidante en los individuos que consumen huevo y evitará la lipoperoxidación ${ }^{(12,13)}$. Esto contribuye a una adecuada asimilación de los lípidos a partir de la yema de huevo sin que se presenten problemas de salud por su consumo.

\section{Efectos en la salud}

El selenio es un oligoelemento involucrado en diversas funciones celulares como antioxidación, refuerzo en el sistema inmune, funciones tiroideas, síntesis de ADN, fertilidad y reproducción ${ }^{(7,10,11,14)}$. Se ha demostrado su función análoga con la enzima glutatión peroxidasa dependiente de selenio (GSH-Px), encargada de destruir los peróxidos tóxicos formados durante el metabolismo intracelular de los lípidos ${ }^{(6,13,15)}$. Los peróxidos tóxicos producen un daño de la membrana celular con posterior salida del citoplasma de la célula y muerte celular $^{(13)}$. La GSH-Px se encarga de proteger a las células del daño causado por los radicales libres, dentro de los factores que modifican los niveles de selenio en el cuerpo se encuentran el sexo, edad, tabaquismo y estado de salud. En fumadores, los requerimientos fisiológicos de selenio aumentan, debido al incremento en la expresión y actividad de las enzimas pulmonares como la glutatión-peroxidasa para eliminar el estrés oxidativo provocado por el cigarrillo ${ }^{(10)}$. Algunos estudios prospectivos han evidenciado ventajas y desventajas acerca de los niveles de selenio. Por una parte, un nivel adecuado puede reducir el riesgo de cáncer, infecciones virales, infertilidad masculina, entre otros. Por otra parte, niveles superiores a los $120 \mu \mathrm{g} / \mathrm{L}$ aumentan el riesgo de diabetes mellitus tipo 2 (DM2) y algunos cánceres $^{(4,6,7)}$. En los pacientes con virus de la inmunodeficiencia humana (VIH) se ha evidenciado que la suplementación con selenio ayuda a reducir el riesgo de contraer algunas infecciones como la tuberculosis (Hazard ratio [HR]: 0,20; intervalo de confianza [IC] $95 \% ; p=0,043)^{(16,17)}$.

De acuerdo con la junta de alimentos y nutrición del Instituto de Medicina de las academias nacionales de Estados Unidos, se recomienda $55 \mu \mathrm{g}$ de selenio/día como cantidad diaria recomendada (RDA) y $400 \mu \mathrm{g}$ de selenio/día como nivel máximo de consumo tolerable para hombres y mujeres entre 19 y 45 años $^{(4)}$. En cuanto a la deficiencia grave de selenio, esta puede debutar como una miocardiopatía endémica (enfermedad de Keshan) o como un tipo de osteoartritis (enfermedad de Kashin-Beck) ${ }^{(4,6)}$. Sin embargo, estas condiciones suelen presentarse en mayor frecuencia cuando se acompañan de deficiencia de yodo o en presencia de toxinas ambientales ${ }^{(4)}$. Por una parte, los síntomas de LOAEL (lowest observed adverse effect level) se han asociado con la pérdida de cabello, dedos de los pies y uñas débiles, así como un olor a ajo en la respiración, caries excesivas y decoloración dental; por otra parte, la toxicidad puede causar síndrome de dificultad respiratoria aguda (SDRA), infarto de miocardio e insuficiencia renal $^{(4,6)}$. La deficiencia ha demostrado ser perjudicial para la salud; sin embargo, altos niveles séricos de este oligoelemento también pueden llegar a ser perjudiciales. Se recomienda que las personas con niveles séricos $>122 \mu \mathrm{g} / \mathrm{L}$ no reciban suplencia de selenio, aunque algunos autores mencionan que se puede mantener una concentración sérica entre 130-150 $\mu \mathrm{g} / \mathrm{L}$, lo cual ha demostrado varios beneficios en la salud sin riesgos adicionales dentro de los que se resaltan efectos inmunoestimulantes, prevención de enfermedades tiroideas autoinmunes y mejoría en la fertilidad ${ }^{(2)}$.

La Tabla 1 muestra el resumen de los beneficios del consumo de huevo de gallina enriquecido con selenio.

\section{Síndrome metabólico}

El síndrome metabólico hace referencia a los factores de riesgo cardiometabólicos dentro de los cuales se encuentra la obesidad abdominal, desregulación de lípidos, presión arterial alta y glucosa alta en ayunas; dichos factores están relacionados directamente con un mayor riesgo de desarrollar enfermedades cardiovasculares y diabetes mellitus ${ }^{(10,18)}$. En este contexto, el selenio ha demostrado ser un antioxidante capaz de reducir los niveles de estrés oxidativo y, de este modo, reducir el síndrome metabólico ${ }^{(4,6,10)}$. Sin embargo, no 
Tabla 1. Resumen de los beneficios del consumo de huevo de gallina enriquecido con selenio

1. El huevo es un alimento de fácil acceso, económico y con bajo impacto calórico en la dieta, lo cual lo convierte en una de las opciones más idóneas para mantener una dieta balanceada y para aporte de selenio.

2. El selenio ha demostrado efectos anticancerígenos, antiinflamatorios y un efecto potencial en la prevención de las enfermedades cardiovasculares. Además de tener un rol importante en la reproducción humana y compromiso del sistema inmune.

3. El selenio influye en el desarrollo del síndrome metabólico mediante la reducción de los niveles de estrés oxidativo debido a su acción antioxidante.

4. En la enfermedad de Parkinson, la disfunción de las selenoproteínas puede influenciar en el desarrollo de esta patología; además, el selenio se ha relacionado con un efecto protector de la bradicinesia y como un adecuado neuroprotector.

5. En enfermedades tiroideas autoinmunes, el selenio ha demostrado su efecto en enfermedades de Hashimoto y Enfermedad de Graves, mejorando los niveles de los anticuerpos antiperoxidasa y disminución de los niveles séricos de $\mathrm{T}_{3}$ y $\mathrm{T}_{4^{\prime}}$ respectivamente.

$\mathrm{T}_{3}$ : triyodotironina; $\mathrm{T}_{4}$ : tiroxina.

se ha observado una asociación estadísticamente significativa entre los niveles de selenio y la prevalencia de los componentes del síndrome metabólico ${ }^{(10)}$.

\section{Enfermedad de Parkinson}

La enfermedad de Parkinson se caracteriza por un daño de las vías dopaminérgicas, principalmente en la sustancia negra, la cual se manifiesta con alteraciones motoras y cognitivas. El selenio es un importante antioxidante en el sistema nervioso central y desempeña un rol fundamental en la neuroprotección ${ }^{(19,20)}$. Las primeras hipótesis fueron realizadas por Cadet, quien sugirió un posible efecto del selenio junto con la vitamina $\mathrm{E}^{(21)}$. Diversos estudios en animales sugieren un efecto protector contra la bradicinesia y el daño del material genético, e inclusive revertir los efectos tóxicos secundarios a la depleción de dopamina ${ }^{(22-24)}$. Sin embargo, en estos ensayos hay ciertas diferencias en cuanto al desarrollo natural de la enfermedad de Parkinson. La deficiencia de selenio puede afectar la función de las neuronas dopaminérgicas; sin embargo, los resultados de diferentes estudios no permiten establecer una relación directa entre el efecto del selenio y el Parkinson ${ }^{(25,26)}$.

Por su parte, el cerebro tiene una alta prioridad para recibir selenio inclusive en el contexto de deficiencia de selenio; además, hay evidencia fuerte que respalda el papel de la disfunción de selenoproteínas en la enfermedad de Parkinson ${ }^{(27)}$. Sin embargo, como se mencionó previamente, la mayoría de estos datos son derivados de estudios en animales y se requiere de más estudios en humanos para determinar el efecto exacto del selenio en la fisiopatología del Parkinson en humanos.

\section{Enfermedad tiroidea}

La glándula tiroides corresponde al órgano con la mayor cantidad de selenio por gramo de tejido ${ }^{(28)}$. Como se mencionó, el selenio es fundamental para el buen funcionamiento del metabolismo de las hormonas tiroideas y la función antioxidante. Los estudios han sugerido que la suplementación con selenio tiene efectos benéficos en enfermedades autoinmunes de la tiroides, especialmente en tiroiditis de Hashimoto, en la que se ha observado disminución de los niveles de anticuerpos antiperoxidasa ${ }^{(29)}$. Otro desorden autoinmune que ha demostrado respuesta a la administración con selenio es la enfermedad de Graves. Los estudios indican que la suplementación con este elemento sumado a la terapia con metimazol en pacientes con hipertiroidismo se asoció con la disminución de los niveles séricos de $\mathrm{T}_{3} \mathrm{y}_{4}$, y con la mejoría clínica de la orbitopatía $^{(30)}$.

En este sentido, el mantenimiento de niveles séricos óptimos de selenio es esencial en la prevención de enfermedad tiroidea, especialmente en pacientes con factores de riesgo para desarrollar enfermedades autoinmunes. El consumo regular de huevo enriquecido con selenio es una fuente costo-efectiva y saludable en aras de mantener concentraciones suficientes de este elemento en la sangre.

\section{CONCLUSIONES}

El selenio es un oligoelemento involucrado en diferentes funciones celulares, dentro de las cuales se encuentra la síntesis del ácido desoxirribonucleico $(\mathrm{ADN})$, refuerzo 
del sistema inmune y antioxidación. El adecuado aporte calórico, bajo costo y fácil acceso en la población colombiana convierten al huevo en un alimento idóneo para ser suplementado con selenio, ya que se podría garantizar un mejor cumplimiento de los requerimientos diarios de este oligoelemento en la población. De esta manera se puede tomar la suplencia de selenio en el huevo de gallina como una medida de prevención y promoción de la salud en la población colombiana.

\section{Fuente de financiación}

Este trabajo tuvo financiación de la Federación Nacional de Avicultores de Colombia (FENAVI).

\section{Conflictos de interés}

Jorge H. Ulloa recibió honorarios por la redacción de este manuscrito. Sebastián Cifuentes, Valentín Figueroa, Emelyn Van Uden y Susana Tafur no tienen conflictos de interés.

\section{Declaración de autoría}

JH. Ulloa participó en el análisis de los resultados, realización y desarrollo de la investigación; S. Cifuentes participó en la redacción del artículo, la concepción, realización y desarrollo de la investigación; V. Figueroa participó en la obtención de los datos y redacción del artículo; E. Van Uden participó en la redacción del artículo y obtención de los datos; S. Tafur participó en la obtención de los datos, redacción del artículo e interpretación de los resultados.

\section{Referencias bibliográficas}

1. Dehghan M, Mente A, Rangarajan S, Mohan V, Lear S, Swaminathan S, et al. Association of egg intake with blood lipids, cardiovascular disease, and mortality in 177,000 people in 50 countries. Am J Clin Nutr. 2020;111(4):795-803. doi: 10.1093/ajcn/nqz348

2. Rayman MP. Selenium and human health. Lancet. 2012;379(9822):1256-1268. doi: 10.1016/S01406736(11)61452-9

3. Lee JH, Paik H-D. Anticancer and immunomodulatory activity of egg proteins and peptides: a review. Poult Sci. 2019;98(12):6505-16. doi: 10.3382/ps/pez381

4. Stoffaneller R, Morse NL. A review of dietary selenium intake and selenium status in Europe and the Middle East. Nutrients. 2015;7(3):1494-537. doi: 10.3390/nu7031494

5. Jiakui L, Xiaolong W. Effect of dietary organic versus inorganic selenium in laying hens on the productivity, selenium distri- bution in egg and selenium content in blood, liver and kidney. J Trace Elem Med Biol. 2004;18(1):65-8. doi: 10.1016/j. jtemb.2004.04.002

6. Huang Y, Wang Q Gao J, Lin Z, Bañuelos GS, Yuan L, et al. Daily dietary selenium intake in a high selenium area of Enshi, China. Nutrients. 2013;5(3):700-10. doi: 10.3390/ nu5030700

7. Mehdi Y, Hornick J-L, Istasse L, Dufrasne I. Selenium in the environment, metabolism and involvement in body functions. Molecules. 2013;18(3):3292-311. doi: 10.3390/molecules 18033292

8. Jaffé W. Selenio, un elemento esencial y tóxico. Datos de Latinoamérica. Arch Latinoam Nutr. 1992;42(2):90-3.

9. Liu H, Yu Q, Fang C, Chen S, Tang X, Ajuwon KM, et al. Effect of selenium source and level on performance, egg quality, egg selenium content, and serum biochemical parameters in laying hens. Foods. 2020;9(1):68. doi: 10.3390/foods9010068

10. Jang H, Morris JS, Park K. Correlates of toenail selenium and its cross-sectional association with metabolic syndrome. J Hum Nutr Diet. 2018;31(5):603-11. doi: 10.1111/jhn.12552

11. Meng T, Liu Y-L, Xie C-Y, Zhang B, Huang Y-Q, Zhang Y-W, et al. Effects of Different Selenium Sources on Laying Performance, Egg Selenium Concentration, and Antioxidant Capacity in Laying Hens. Biol Trace Elem Res. 2019;189(2):548-55. doi: 10.1007/s12011-018-1490-z

12. Wang D, Dinh QT, Anh TTT, Zhou F, Yang W, Wang M, et al. Effect of selenium-enriched organic material amendment on selenium fraction transformation and bioavailability in soil. Chemosphere. 2018;199:417-26. doi: 10.1016/j.chemosphere.2018.02.007

13. Morales LR, García MAR, Rosiles MR. Correlación del contenido de selenio en el alimento con el de la yema de huevo en gallinas de postura. Vet México. 2001;32(3):225-7.

14. Chinrasri O, Chantiratikul P, Thosaikham W, Atiwetin P, Chumpawadee S, Saenthaweesuk S, et al. Effect of seleniumenriched bean sprout and other selenium sources on productivity and selenium concentration in eggs of laying hens. AsianAustralasian J Anim Sci. 2009;22(12):1661-6. doi: 10.5713/ ajas.2009.90220

15. Fisinin VI, Papazyan TT, Surai PF. Producing seleniumenriched eggs and meat to improve the selenium status of the general population. Crit Rev Biotechnol. 2009;29(1):18-28. doi: 10.1080/07388550802658030

16. Rayman MP. Selenium intake, status, and health: a complex relationship. Hormones. 2020;19(1):9-14. doi: 10.1007/ s42000-019-00125-5

17. Campa A, Baum MK, Bussmann H, Martinez SS, Farahani $\mathrm{M}$, van Widenfelt $\mathrm{E}$, et al. The effect of micronutrient supplementation on active TB incidence early in HIV infection in Botswana. Nutr Diet Suppl. 2017;2017(9):37-45. doi: 10.2147/NDS.S123545 
18. Yaroshenko FO, Dvorska JE, Surai PF, Sparks NHC. Seleniumenriched eggs as a source of selenium for human consumption. Appl Biotechnol Food Sci Policy. 2003;1(1):13-23.

19. Maldonado PD, Pérez-De La Cruz V, Torres-Ramos M, SilvaIslas C, Lecona-Vargas R, Lugo-Huitrón R, et al. Seleniuminduced antioxidant protection recruits modulation of thioredoxin reductase during excitotoxic/pro-oxidant events in the rat striatum. Neurochem Int. 2012;61(2):195-206. doi: 10.1016/j.neuint.2012.05.004

20. Lu Z, Marks E, Chen J, Moline J, Barrows L, Raisbeck $\mathrm{M}$, et al. Altered selenium status in Huntington's disease: Neuroprotection by selenite in the N171-82Q mouse model. Neurobiol Dis. 2014;71:34-42. doi: 10.1016/j. nbd.2014.06.022

21. Cadet JL. The potential use of vitamin $\mathrm{E}$ and selenium in Parkinsonism. Med Hypotheses. 1986;20(1):87-94. doi 10.1016/0306-9877(86)90089-7

22. Ellwanger JH, Franke SIR, Bordin DL, Prá D, Henriques JAP. Biological functions of selenium and its potential influence on Parkinson's disease. An Acad Bras Ciênc. 2016;88(supl 3):1655-1674. doi: 10.1590/0001-3765201620150595

23. Khan HA. Selenium partially reverses the depletion of striatal dopamine and its metabolites in MPTP-treated C57BL mice. Neurochem Int. 2010;57(5):489-491. doi: 10.1016/j. neuint.2010.06.020

24. Zafar KS, Siddiqui A, Sayeed I, Ahmad M, Salim S, Islam F. Dose-dependent protective effect of selenium in rat model of Parkinson's disease: neurobehavioral and neuroche- mical evidences. J Neurochem. 2003;84(3):438-46. doi: 10.1046/j.1471-4159.2003.01531.x

25. Aguilar MV, Jiménez-Jiménez FJ, Molina JA, Meseguer I, Mateos-Vega, González-Muñoz MJ, et al. Cerebrospinal fluid selenium and chromium levels in patients with Parkinson's disease. J Neural Transm. 1998;105(10-12):1245-51. doi: $10.1007 / \mathrm{s} 007020050127$

26. Youne-Mhenni S, Aissi M, Mokni N, Boughammoura-Bouatay A, Cheberl, Frih-Ayed M, et al. Serum copper, zinc and selenium levels in Tunisian patients with Parkinson's disease. Tunis Med. 2013;91(6):402-5.

27. Zhang X, Liu R-P, Cheng W-H, Zhu J-H. Prioritized brain selenium retention and selenoprotein expression: Nutritional insights into Parkinson's disease. Mech Ageing Dev. 2019;180:89-96. doi: 10.1016/j.mad.2019.04.004

28. Ventura M, Melo M, Carrilho F. Selenium and Thyroid Disease: From Pathophysiology to Treatment. Int J Endocrinol. 2017;2017:1297658. doi: 10.1155/2017/1297658

29. Gärtner R, Gasnier BCH, Dietrich JW, Krebs B, Angstwurm MW. Selenium supplementation in patients with autoimmune thyroiditis decreases thyroid peroxidase antibodies concentrations. J Clin Endocrinol Metab. 2002;87(4):1687-91. doi: 10.1210/jcem.87.4.8421

30. Wang L, Wang B, Chen SR, Hou X, Wang XF, Zhao SH, et al. Effect of selenium supplementation on recurrent hyperthyroidism caused by Graves' disease: a prospective pilot study. Horm Metab Res. 2016;48(9):559-64. doi: 10.1055/s-0042110491 